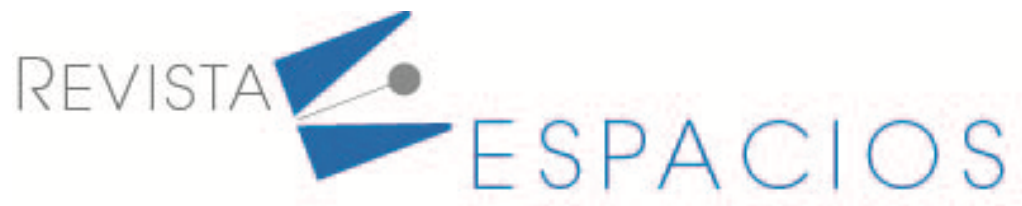

\title{
Diagnósticos y planes de igualdad en empresas vascas: de la teoría a la práctica
}

\author{
Diagnosis and equality plans in Basque companies: from theory to practice
}

\author{
AURREKOETXEA-CASAUS, Maite \\ MARTÍNEZ-MORÁN, Pedro César \\ DÍEZ, Fernando ${ }^{1}$
}

\begin{abstract}
Resumen
En este artículo se evalúa la potencial efectividad de los planes de igualdad de las empresas vascas a través de un análisis cualitativo de su contenido tratando de identificar, desde el punto de vista del diseño del plan, las áreas prioritarias y la congruencia entre objetivos y medidas. Los principales resultados evidencian un bajo interés en las principales barreras para la igualdad en el desempeño, como medidas de promoción o de selección, así como el carácter propositivo de los planes analizados.

Palabras clave: planes de igualdad; genero; normativa; empresa
\end{abstract}

\begin{abstract}
This article evaluates the potential effectiveness of the equality plans of Basque companies through a qualitative analysis of their content, trying to identify, from the point of view of the plan design, the priority areas and the congruence between objectives and measures. The main results show a low interest in the main barriers to equality in performance, such as promotion or selection measures, as well as the propositional nature of the plans analyzed.
\end{abstract}

Key words: equality plans; gender; regulations; company

\section{Introducción}

El vocabulario que ha generado la igualdad de oportunidades se ha visto alimentado por conceptos tales como: barreras, techo de cristal, suelo pegajoso, solidaridad de género, laberinto, tubería con fugas, discriminación salarial o escaleras de ascenso. La ruptura de ciertos clichés parece haberse conseguido. En 2021, Rachael Blackmore se ha convertido en la primera dama que gana el Grand National (de Miguel, 2021). Anne Panther, árbitro de la Euroliga de baloncesto, confesó que es remunerada con el mismo salario que sus colegas masculinos (Dazn, 2021). Son hitos que demuestran que la igualdad efectiva dista mucho de ser real mientras todos estos casos sean titulares de prensa. Sin embargo, el Global Gender Gap (World Economic Forum, 2021) señala que, al menos el $41 \%$ de los profesionales en posiciones senior son mujeres, a pesar de la persistencia del techo de cristal en Estados Unidos, Italia, Gran Bretaña o Países Bajos. Según el World Bank Group (2019), a las mujeres solo se les reconoce el $75 \%$ de los derechos de los que gozan los hombres, y 56 países no habían dado ningún paso en pos de la igualdad durante el período de diez años que abarca su análisis.

${ }^{1}$ Universidad de Deusto, España. fdiez@deusto.es 
En el ecosistema empresarial, existe en las organizaciones una infrarrepresentación femenina en posiciones de manager o de alta dirección con respecto a sus colegas masculinos (Maitland, 2015; Robinson, 2018; Kendall, 2018; Peluso et al. 2019; Huang et al. 2019; McKinsey, 2015; Kaiser y Wallace, 2016). El origen de esta asimetría obedece a un componente multifactorial: preferencia por seleccionar a personas del mismo sexo (Molero, 2009), estereotipos de género (Escot et al. 2008; Sarrió et al. 2002; Hopkins y O’Neil, 2007; Collins, 2005), oportunidades de desarrollo (Barberá et al, 2002) o problemas derivados de la conciliación de la vida laboral y personal (Martín, 2005; Nieto y Hernández, 2007; López-Ibor, 2008). En todo caso, las derivaciones de todas las causas implicadas originan que, a finales de 2019, y en las empresas que superaban los 500 millones de capitalización bursátil, el porcentaje de altas directivas era del 17,7\%, siendo algo menor, el 13,4\%, en las compañías por debajo de los 500 millones de capitalización. Todo ello por debajo del 40\%, objetivo fijado para 2022 por las sociedades cotizadas (CNMV, 2021). De hecho, a finales de 2020, las mujeres ocupan un $27,7 \%$ de los puestos de los consejos de administración de las empresas del IBEX 35 (INE, 2021).

Existen varias vías para incentivar la mayor presencia de las mujeres en los puestos de dirección, altos y medios, de las empresas. Desde la mejora de los procesos de selección y promoción de talento al establecimiento de códigos de buenas prácticas, los programas para prevenir acoso psicológico y sexual, la formación en género o el cumplimiento de la normativa vigente en cada caso. El Consejo de Europa ha generado instrumentos jurídicos y de orientaciones normativas (Ministerio de Asuntos Exteriores y de Cooperación, 2021) para alcanzar el progreso en la igualdad entre hombres y mujeres.

La legislación aporta un respaldo, proporcionado por la obligatoriedad de la medida, un castigo, por las sanciones derivadas de incumplimiento y un señalamiento social, a las empresas que incumplan la legislación, circunstancia que afecta a su employer branding, a su negocio y a sus relaciones. Y, sobre todo, muestran el camino a seguir para evitar la discriminación y alcanzar la igualdad de oportunidades de las mujeres en su desarrollo profesional.

Las cuestiones ligadas a la política de igualdad se pueden salvar desde la apuesta por la norma que propicie la aceptación de la diversidad (Dobbing y Kelly, 2007). El obligado cumplimiento que supone la normativa laboral sirve de impulso para que las empresas incorporen directoras y adopten políticas de diversidad (Zhang, 2020), como elemento capital para las nuevas decisiones en materia de adquisición y promoción de talento.

El artículo 14 de La Constitución Española (1978) destaca que no puede prevalecer discriminación alguna. Con anterioridad a la Ley Orgánica de 2007, sendas órdenes ministeriales (de España, 2005) aprueban: 1) adoptar medidas que favorezcan la igualdad entre mujeres y hombres y 2) el Plan para la igualdad de género en la Administración General del Estado. De la sensibilidad y el enfoque general, se ha transitado a normativas y exigencias concretas, de modo que España, en la actualidad, suma a su propia legislación, los requerimientos de carácter voluntario para promover la diversidad de género (Kersley et al., 2019). Se añaden, en ambos casos, obligación y consejos, la opción de cumplir con determinados niveles de cuotas, la obligatoriedad de planes de igualdad en las empresas y del registro retributivo, éste último en vigor desde el 14 de abril de 2021.

El plan de Igualdad constituye una herramienta compuesta por un conjunto estructurado de medidas que persigue un doble objetivo: 1) alcanzar la igualdad de trato y de oportunidades entre hombres y mujeres y 2) la ausencia de discriminación por razón de sexo. La estructura de un Plan de Igualdad para las empresas viene definida en la guía desarrollada por el Ministerio de Igualdad a tal efecto (2021) y se soporta en cinco hitos: 1) Puesta en marcha del proceso de elaboración del plan; 2) Diagnóstico 3) Diseño, aprobación y registro del plan de igualdad; 4) Implantación y seguimiento del Plan y 5) Evaluación del Plan de Igualdad. La propia guía supone la aplicación de los principios normativos sobre lgualdad, propone las actuaciones a realizar y proporciona los instrumentos a las empresas para implantarla, según sea el tamaño de las organizaciones.

Los Planes de Igualdad en España se soportan legalmente según se expresa en la tabla 1: 
Tabla 1

Legislación y acuerdos aplicables

en España en materia de Igualdad

\begin{tabular}{lc}
\hline \multicolumn{1}{c}{ Legislación } & Fecha \\
\hline Ley Orgánica 3/2007 para la igualdad efectiva de mujeres y hombres & 22 marzo 2007 \\
$\begin{array}{l}\text { Real Decreto Legislativo 2/2015, texto refundido de la Ley del Estatuto de los } \\
\text { Trabajadores }\end{array}$ & 23 octubre 2015 \\
$\begin{array}{l}\text { Real Decreto Ley 6/2019, de medidas urgentes para garantía de la igualdad de trato y } \\
\text { de oportunidades entre mujeres y hombres en el empleo y la ocupación }\end{array}$ & 1 marzo 2019 \\
Acuerdo por la igualdad efectiva entre mujeres y hombres en el trabajo & 30 julio 2020 \\
\hline
\end{tabular}

Nota: elaboración propia a partir de BOE, Ministerio de Igualdad.

La tabla 2 detalla la fecha límite en la que las empresas deben de haber definido un plan de igualdad en función del tamaño de su plantilla.

Tabla 2

Fecha planes de igualdad

por tamaño de empresa

\begin{tabular}{lcc}
\hline \multicolumn{1}{c}{ Empresas } & Fecha Plan lgualdad & Observaciones \\
\hline Más de 250 trabajadores & 22 marzo 2007 & Así como empresas en las que el convenio colectivo lo exprese \\
Más de 150 trabajadores & 7 marzo 2020 & \\
Más de 100 trabajadores & 7 marzo 2021 & \\
Entre 50 y 100 trabajadores & 7 marzo 2022 & \\
Menos 50 trabajadores & & Registro retributivo a partir de 14 abril 2021 \\
\hline
\end{tabular}

Nota: elaboración propia a partir de datos de BOE, Ministerio de Igualdad

Existen, por tanto, varios años en los que las empresas muestran evidencias de la implantación de los planes de igualdad. Sin embargo, no es fácil encontrar resultados sobre el impacto de la implantación de los planes sobre la igualdad real. El planteamiento que se realiza desde esta investigación se apoya en la idea de Gelambi-Torrell (2015) quien identifica el diseño de los planes de igualdad como una de las principales debilidades, por el riesgo de convertirse en declaraciones de buenos propósitos sin llegar a acciones concretas. Lo que reducirá a los planes a una herramienta de sensibilización y de movilización de la conciencia en materia de igualdad.

El estado incipiente de implantación de los planes de igualdad junto con la opacidad de las empresas dificulta una imagen real de la situación y hace que el interés de esta investigación se centre en conocer cuáles son las áreas de interés con respecto a la igualdad a través del análisis de casos seleccionados. Conscientes de la dificultad que esto entraña, esta investigación, se propuso explorar desde el diseño de los planes de igualdad, la congruencia entre los objetivos y las medidas a implantar de tal forma que mostrara la potencialidad de los planes como instrumentos del cambio en la empresa.

No se trata, por lo tanto, de presentar los resultados de los planes, puesto que, en la gran mayoría de los casos, ni las propias empresas establecen indicadores de resultado sobre la implantación del plan, sino más bien de representar las potencialidades de un plan en función del diseño. De esta forma se puede visualizar el foco puesto por los responsables de plantear el Plan y representar el interés real de las empresas por la igualdad.

La estructura seguida en la investigación quedó establecida en los siguientes apartados. En primer lugar, un análisis teórico de las principales barreras de género en la empresa, con especial interés en la promoción y selección como elemento discriminatorio. A continuación, se plantea la estrategia metodológica seguida, basada en un análisis de contenido de planes de igualdad de cinco empresas seleccionadas. En tercer lugar, se recogen los principales resultados del estudio. Entre ellos, destacar que en los planes de igualdad analizados se observa 
una incongruencia entre los objetivos generales y específicos planteados y el peso que las medidas para alcanzar esos objetivos se diseñan en dichos planes. Para finalizar, se presentan las conclusiones derivadas de los mismos, en la que se resalta que la existencia que una preocupación por tratar de paliar la presencia de la discriminación formal e informal en los procesos de promoción en las empresas participantes en el estudio, pero que no se concreta en medidas específicas.

\subsection{Marco de referencia}

En España, al comienzo de la década de los ochenta del siglo pasado, se produce un incremento en la incorporación de la mujer al mercado laboral (Cebrián y Moreno, 2008). Esta integración ha permitido la aportación de talento femenino. Sin embargo, no es óbice para seguir exhibiendo una serie de barreras externas e internas.

Entre las dificultades para la promoción de la mujer en las organizaciones, el Esade Gender Monitor (2021) señala a la mayor inversión del sector masculino en crear y mantener redes de apoyo, las dificultades para la conciliación laboral y de crianza de los menores y otras dificultades más vinculadas con la cultura organizativa como, por ejemplo, la asunción de que la mujer tiene un compromiso menor con los objetivos de la empresa.

Estas dificultades se asientan sobre la teoría del capital cultural, cuyo núcleo explicativo encaja con el propio proceso de socialización humano: hogar y escuela como vehículos primarios. En este sentido, puede entenderse que determinadas asimetrías socioeconómicas respecto a los logros educativos entre mujeres y hombres se encuentran en una correlación positiva entre el hábito (Bourdieu, 1979) y la transición a la educación superior (Edgerton y Roberts, 2014; Roksa y Robinson, 2017). Autores como Wilson y Urick (2021) atribuyen la disparidad en el progreso profesional de mujeres y hombres a que aquellos con más habilidades en la resolución de problemas han tenido un mayor acceso a recursos normativos y a las redes sociales alienadas con los principios académicos.

A los argumentos basados en los géneros se suman dos cuerpos doctrinales: uno relacionado con los estereotipos sociales y otro con la masculinización de ciertas profesiones. En el primer caso, una de las principales barreras se encuentra en la progresión de las mujeres a puestos directivos basada en el paradigma de Schein (1973) "think manager-think male". Así lo corroboran García-Ael et al. (2012), que también subrayan que realizar el mismo trabajo no elimina los estereotipos de género, como arguye la teoría del rol social (Eagly, 1987).

Por otro lado, la teoría de la masculinización de ciertas profesiones hace pensar en que las mujeres se sienten menos atraídas por profesiones donde se visualizan mayores dificultades o un clima poco favorable para su desarrollo profesional y personal. Wajcman (2006) se cuestiona si la tecnología tiene sexo y argumenta que los avances en dicho campo están provocando una realineación de la relación mujer-máquina y, por ende, del papel del hombre, pretendidamente más expuesto a dicha relación. Esa relación entre tecnología y hombre les lleva a elegir este campo, tal y como plantean McKinney et al. (2008) que, por otro lado, no encontraron diferencias significativas entre hombres y mujeres en este ámbito profesional, salvo que el equilibrio de trabajo y familia conduce a la mujer a abandonar y buscar otras oportunidades profesionales. Goyette y Mullen (2006) matizan que la diferencia de elección de grado entre hombres y mujeres radica en la remuneración: los hombres tienden a elegir a aquellos grados que les conduzcan a profesiones mejor remuneradas.

A las barreras exógenas que impiden el progreso de la mujer en las organizaciones, se le unen aquellas propias de cada empresa, y en las que subyace una cultura corporativa exenta de igualdad y, por tanto, de una asimetría que encarece el talento, al mirar o apostar en una sola dirección. Beltrán et al. (2018) establece cuatro limitaciones de las mujeres directivas españolas. A la ya conocida dificultad de conciliar (Vega et al., 2007) y la escasa corresponsabilización de los hombres en el ámbito doméstico, deben unirse la falta de implicación de la 
alta dirección que provoca una falta de mujeres referentes en dichas posiciones y que se apoya en una inadecuada cultura organizacional que estimule la promoción de las mujeres.

Hacohen et at. (2021) subrayan que son las mujeres las que doblan a los hombres en la proporción de preferir trabajos con jornada reducida, flexibilidad de horarios, etc, circunstancias que se amplifican en el caso de tener hijos, y que esa condición de ser madre con hijos, ha dejado un estereotipo respecto a decantarse por la familia en lugar de por su trabajo. Y este hecho se acrecienta en las mujeres que, debido al suelo pegajoso, se encuentran atrapadas en niveles de renta bajo (Ministerio de Sanidad, Servicios sociales e Igualdad, 2016), y con escasas posibilidades de salir de ese círculo vicioso.

Las consecuencias negativas para la mujer vienen de la parada de su carrera profesional (Hewlett y Luce, 2005) o del abandono de la misma (Belkin, 2004; Wallis, 2004). Previamente, en la incorporación a la empresa, los sistemas sesgados favorecen al hombre (Biedma-Ferrer, 2017; Doldor et al. 2012; Tienari et al. 2013). Y la cultura organizativa que se enriquece gracias a la conjunción de los ámbitos formal e informal, tiene, precisamente en este último a otra barrera para las mujeres al excluirlas de las redes informales (Hopkins y O’Neil, 2007).

A pesar de que los datos muestran una evolución social hacia el incremento de mujeres en posiciones directivas, los cambios culturales y educativos son un factor complejo que requiere de instrumentos y de planes de acción, donde los poderes públicos deben de auxiliar a las empresas en esta tarea. Estos factores se ven alimentados por la falta de criterios claros de selección en igualdad de oportunidades, la ausencia de transparencia en la retribución de los empleados o de criterios claros de promoción (Salinas, y Bagni, 2017; Correll, 2017; Trotter et al., 2017; Morgan, 2019; Bennedsen et al., 2019; Bryant, 2017). No parece que haya otra fórmula para evitar la fuga de talento femenino en las organizaciones, salvo apostar por políticas de flexibilidad (Chinchilla y León, 2007) y de promoción (Lerer, Z., y Ben-Eliyahu, H. (2019), en el marco de una cultura dispuesta a ello.

La transición entre posiciones en las organizaciones viene dada tanto por los mecanismos de adquisición de talento externo como por los de promoción del talento interno. Respecto a estos últimos, existen diversas barreras (Martín de la Torre, 2017; Baños, 2018; Hoops et al., 2018; Silver, 2019) ILO (2019) considera que la presencia o no de las mujeres en posiciones directivas tiene que ver con las opciones que tienen de promocionar y que los procesos y los programas de promoción se definen por criterios estereotipados. Kellerman y Rhode (2007) afirman que el sendero de la promoción para la mujer se asemejaría más a un laberinto.

En los procesos de adquisición de talento, los managers pueden decantarse sesgadamente, entre candidatos similares, por aquellos masculinos al ser considerados estos más competentes (Ro, 2021). Los estereotipos forman parte del proceso cognitivo humano y la dificultad radicaría, no en lo que significan, sino en lo que irradian respecto a cómo se comportan los así señalados (Molero et al., 2009). Circunstancia que afectaría a las mujeres en su proceso de desarrollo en las compañías, dada que la promoción está asociada a la asunción de mayores responsabilidades $y$, por tanto, a liderar equipos y a gestionar recursos. El modelo a imitar del que parten es el masculino (Munduate, 2003). Y no solo es la referencia para definir el modelo de liderazgo. De las escuelas universitarias salen muchos más egresados masculinos que femeninos para surtir de talento a distintos sectores tales como automoción, industria manufacturera, energía o tecnología (Krivkovich et al., 2016). La brecha STEM se explicaría desde ámbitos biológicos, culturales y psicológicos (Inga y Tristán, 2020). Dicha brecha, circunscrita a un conjunto de saberes y conocimientos, soportaría la menor presencia de talento femenino en la entrada a las organizaciones y, por tanto, menor posibilidad de promocionar en el futuro.

La evaluación del desempeño mecaniza y avala el desarrollo del talento. ILO (2019) advierte que algunos documentos de evaluación del desempeño tienen un sesgo masculino. Por añadidura, la solidaridad de género (Fajak y Haslam, 1998) tiende a favorecer a los del mismo género cuando se trata de analizarles. Circunstancia 
que, para las mujeres, funciona como defensa frente a las inferiores posiciones que han ocupado en las organizaciones, y que favorece a los hombres porque ocupan esos lugares en el organigrama en mayor número.

McKinsey (2007) advertía que ciertas preguntas en los procesos de evaluación de desempeño, como aquellas referidas a movilidad geográfica o disponibilidad, penalizaban a las mujeres puesto que son ellas las que mantenían la doble carga: trabajo y gobierno del hogar. $Y$ esa dificultad propiciaba que renunciaran a su carrera o que optasen por una jornada flexible.

Los sistemas de promoción deben de basarse en pruebas objetivas de competencias y habilidades (Fundación Factor Humá, 2010). Se evitaría el efecto tijera (Martínez Ruiz et al., 2018). Además, conviene definir la forma en que se definen las vías de promoción, la movilidad potencial entre puestos y las herramientas de evaluación que se emplean (Lerer, y Ben-Eliyahu, 2019), con una adecuada formación para evaluador y evaluado. Escapa y Ten (2010) se apoyan en las distintas etapas de la atracción y desarrollo del talento, para afianzar la posición de las mujeres y apostar por su promoción con un enfoque estructurado. Bortz (2018) refleja casos de uso de la selección ciega como elemento generador de diversidad. PriceWaterhouseCoopers (2020) ha incorporado ejemplos de buenas prácticas para la igualdad en la empresa. El talento y su creciente necesidad se convertirán en aliados de las mujeres debido a la presión que tienen las grandes corporaciones por incorporar a las personas con el mejor potencial (Peluso et al., 2019), sin discriminación de género alguna.

\section{Metodología}

Con el objetivo de tratar de identificar, trazar y comprender la congruencia entre los objetivos generales y específicos con las medidas diseñadas para la consecución de dichos objetivos se planteó la técnica de análisis de contenido que permite interesarse por dos "especies de realidad: la realidad de los datos, lo que los Planes de Igualdad arrojen, y la realidad de lo que el investigador quiere conocer en este, la potencial eficacia de los Planes de igualdad. Obviamente la realidad de la igualdad en la empresa no puede ser observada directamente, es por esto, que Krippendorff (2002) señala que la técnica de análisis de contenido permite clarificar lo que al investigador le interesa conocer de verdad y no puede ser observado directamente, es decir, en el caso de los planes de igualdad, analizar los documentos concretos, posibilita visualizar las áreas de interés de las empresas en materia de igualdad y la consideración de los planes de igualdad como instrumentos para el cambio en la empresa.

\section{Etapa 1: selección de los casos}

Esta investigación se ha realizado en colaboración con la Asociación HAB (PWN) que cuenta con un grupo de empresas (Think Tank) que realiza la función de grupo-motor para las iniciativas de la Asociación en materia de igualdad en las empresas del País Vasco. Son, por lo tanto, empresas con un alto grado de implicación en materia de igualdad en la empresa, a las que se les pidió el acceso a los planes de igualdad, con objeto de conocer donde las empresas ponen el foco en las áreas de actuación en materia de igualdad y la congruencia entre los objetivos diseñados en los planes y las medidas a implantar.

De las diez empresas que forman parte del grupo-motor, seis de ellas se ofrecieron a participar en dicha investigación por lo que la muestra resultante quedo configurada tal y como se refleja en la Tabla 3.

El producto de su participación dio como resultado la revisión de 8 documentos (ver Tabla 3). Excepto una, el resto se encuentran ya en el desarrollo del II Plan de Igualdad, con diferentes metodologías aplicadas para la realización del diagnóstico y del establecimiento de objetivos generales y específicos, lo que dificultó la comparación de documentos. 
Tabla 3

Relación de empresas anonimizadas participantes del estudio

\begin{tabular}{ccc}
\hline Id. Entidad & Sector Actividad & Documentos analizados \\
\hline TIGER-BROTHERS & Distribución Logística & I Plan Igualdad (2015-2018)+ II Plan Igualdad (2019-2021) \\
LION - KITS & Servicios financieros y bancarios & II Plan de Igualdad (2016-2018) \\
JAGUAR -TIME & Industria y Distribución & II Plan de Igualdad 2019-2022 \\
PANTHER -ZONE & Comercio & II Plan de Igualdad 2013-2017 \\
ELEPHANT- ARTIST & Servicios Avanzados & II Plan de Igualdad (2018-2022) \\
DOLPHIN -TEC & Industria y Distribución & II Plan (2018-2022)
\end{tabular}

Nota: elaboración propia

\section{Etapa 2: Selección categorías de análisis y ciclos de codificación}

Para el análisis de los planes se utilizó el software de análisis cualitativo de datos Maxqda. El primer ciclo de codificación se apoyó en las diez áreas a desarrollar en los planes de igualdad establecidas por la Ley Orgánica para la igualdad efectiva de mujeres y hombres (2007) y que se concretan en: 1) Compromiso con la igualdad 2) Conciliación 3) Reclutamiento, selección y contratación 4) Salud laboral 5) Promoción profesional 6) Acoso sexual 7) Formación continua 8) Violencia de género 9) Igualdad retributiva y 10) Comunicación y sensibilización.

En un segundo ciclo de codificación, los documentos analizados fueron tratados en dos momentos: el primer momento de Diagnóstico y el segundo momento, el Plan propiamente dicho con la presentación de los objetivos generales, específicos, medidas, indicadores, recursos, cronograma y presupuesto. En este momento se recogieron con especial interés los objetivos generales y específicos en cada de unas Áreas establecidas, para que en un tercer momento de la codificación fueran seleccionadas las medidas establecidas para cada una de las áreas y sus objetivos.

\section{Etapa 3. Procedimiento y análisis}

Con la información obtenida gracias a la codificación de los datos, se establecieron dos niveles de análisis. EI primero, en el que se estudiaron temáticamente los objetivos generales y específicos de los planes analizados. Y un segundo nivel de análisis, en el que se realizó un contraste entre objetivos generales y específicos y a su vez con las medidas planteadas, previamente codificadas, para la consecución de dichos objetivos.

Para la discusión se interpretaron los resultados obtenidos del contraste inter y intra con la literatura revisada, elaborando las principales reflexiones y conclusiones sobre los resultados.

\section{Resultados}

El análisis de contenido realizado arrojó los siguientes resultados. Del contraste entre las áreas de mejora y los puntos fuertes que se presentan en los Diagnósticos preliminares a la concreción del Plan, las empresas proponen, como primer resultado del análisis, mejorar su compromiso con la igualdad, muy unido a la necesidad de mejora en la comunicación y sensibilización en la empresa. El segundo resultado del análisis plantea una incongruencia entre los objetivos y las medidas planteadas en los Planes. En tercer lugar, los Planes quedan sin concreción de recursos y temporalidad.

\section{Resultado 1: Puntos Fuertes versus Áreas de Mejora en el Diagnóstico}

Del análisis de los Diagnósticos realizados previos a la concreción del Plan en objetivos generales, específicos y medidas, los planes de las empresas analizadas señalan que los puntos fuertes en materia de Igualdad se centran: 
en el compromiso por la igualdad, el acceso, selección y contratación y la promoción. Son Áreas sobre las que se está trabajando y sobre las que se tiene interés en abordar, dada la conciencia que tienen los planificadores en el poder de estas áreas para el equilibrio entre mujeres y hombres en las empresas.

Figura 1

Visualización del peso de la codificación en las 10 áreas analizadas en el diagnóstico del plan de igualdad

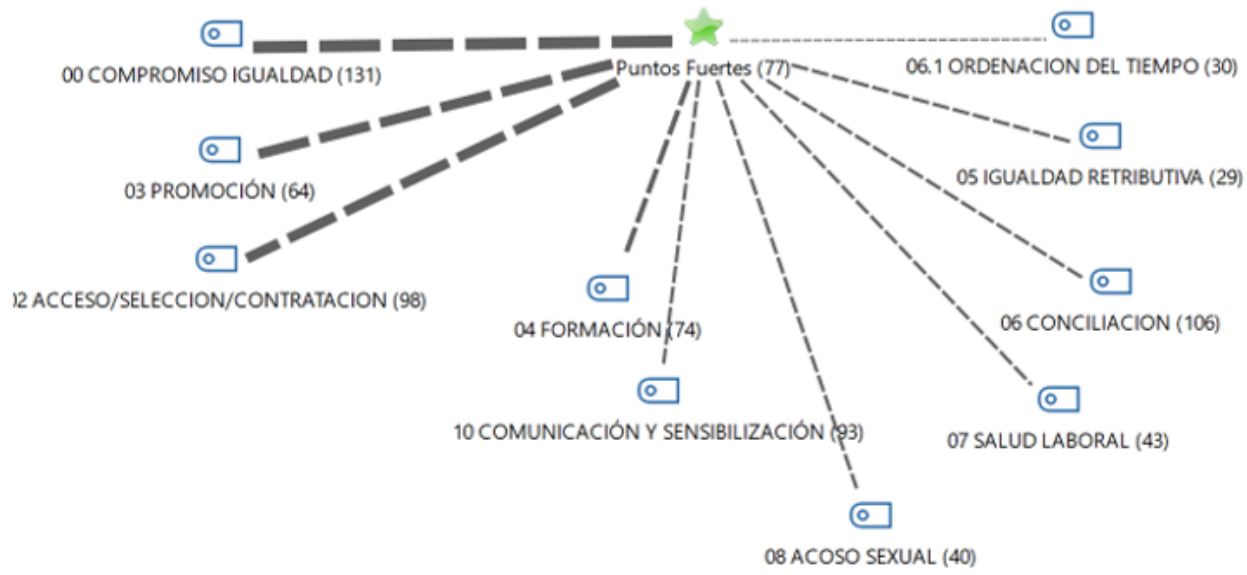

Nota: elaboración propia

Con respecto a las Áreas de Mejora detectadas en el Diagnóstico preliminar, los planes de las empresas analizadas orientan la mejora hacia aquellas áreas sobre las que es previsible plantear objetivos y medidas alcanzables a corto plazo, sin que exijan grandes esfuerzos organizativos. De nuevo el compromiso con la Igualdad adquiere relevancia y, en segundo lugar, el acceso, selección y contratación es vista por las entidades como un área sobre la que poner el foco para mejorar la situación de igualdad en la empresa (ver Figura 2). Por ejemplo, la promoción interna exigiría herramientas y mecanismos de mayor calado.

Figura 2

Incongruencia objetivos generales y específicos con las medidas plantadas en los planes

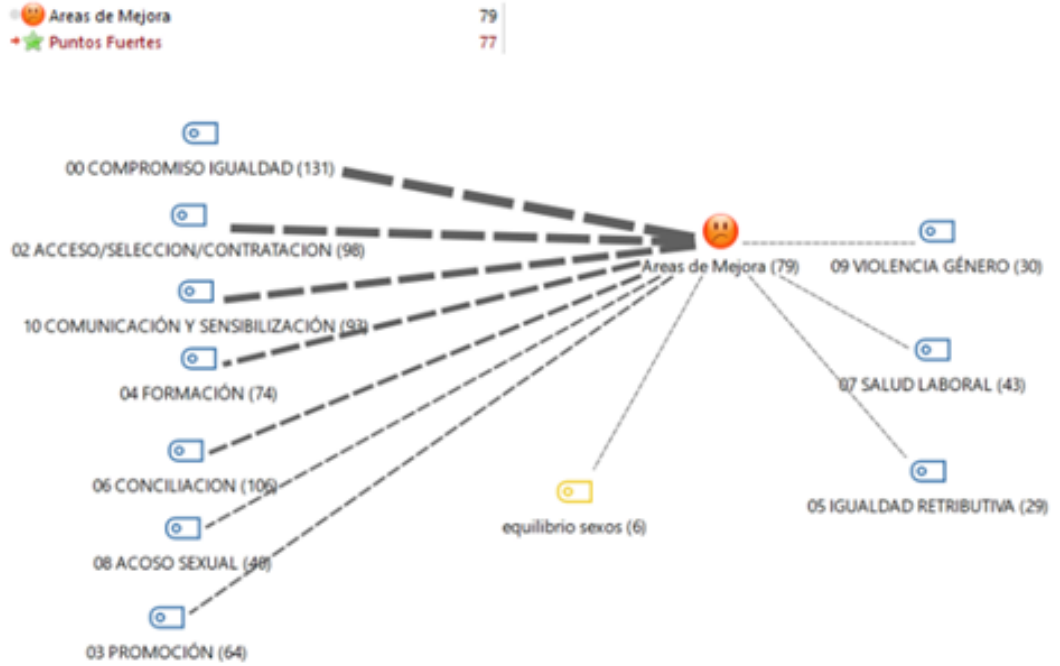

Nota: elaboración propia 


\section{Resultado 2: Incongruencia entre objetivos generales y específicos con las medidas diseñadas}

Un análisis más en detalle del peso diferencial de las Áreas en los planes, arroja una preocupación por el área 10 relativa a la Comunicación y Sensibilización. Esto podría indicar que las entidades analizadas están en un modelo "incipiente" de desarrollo de la Igualdad de la empresa al tratar de comenzar por aquellos objetivos y medidas de más fácil desarrollo e implantación. Son todos objetivos y acciones orientados a conseguir una mejor comunicación interna y externa que visibilice la preocupación e interés de la empresa por la igualdad en la empresa (ver tabla 4): Comunicación y sensibilización, con un peso de codificación (27) compromiso con la igualdad (20) y conciliación (14).

Áreas como el acceso, selección, contratación y promoción están escasamente representadas en los planes, junto con el hecho de que no exista ningún objetivo ni medida orientada a la igualdad retributiva, puede estar indicando que los planes de igualdad comienzan por aquellas áreas que tienen una mejor aplicación.

\section{Tabla 4}

Visualización de coocurrencia de códigos de Áreas de Igualdad y Objetivos Generales

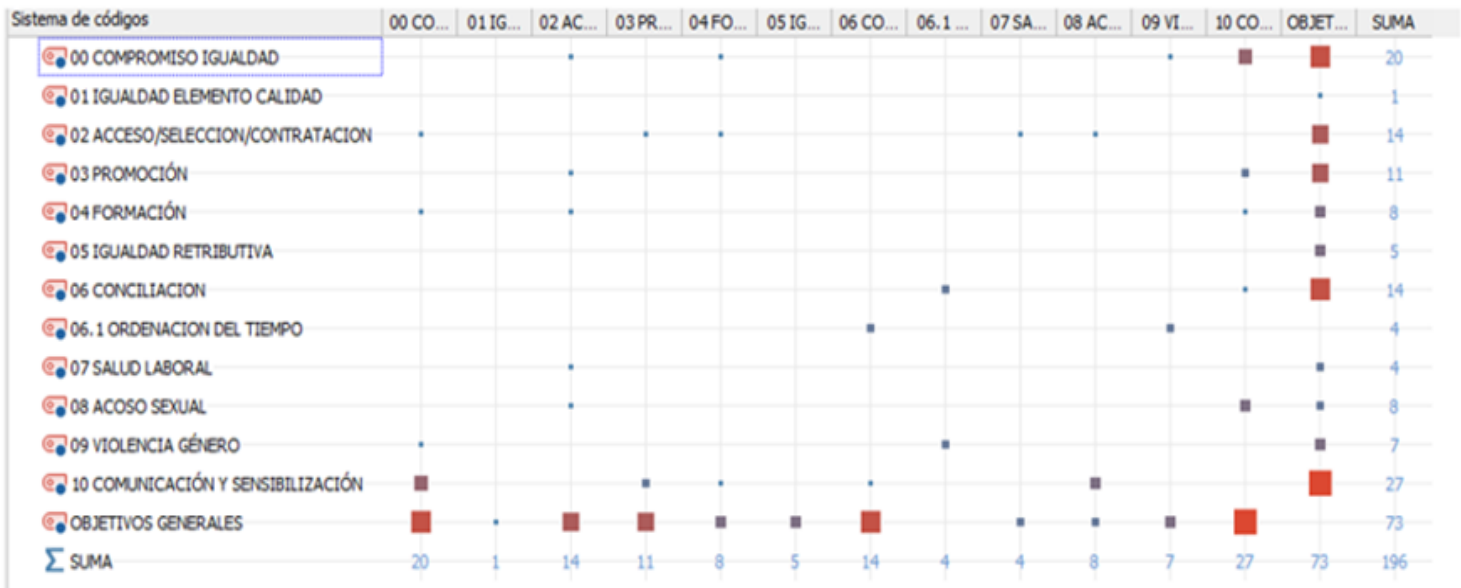

Nota: elaboración propia

Tabla 5

Visualización de coocurrencia de códigos de áreas de igualdad y objetivos específicos/operativos

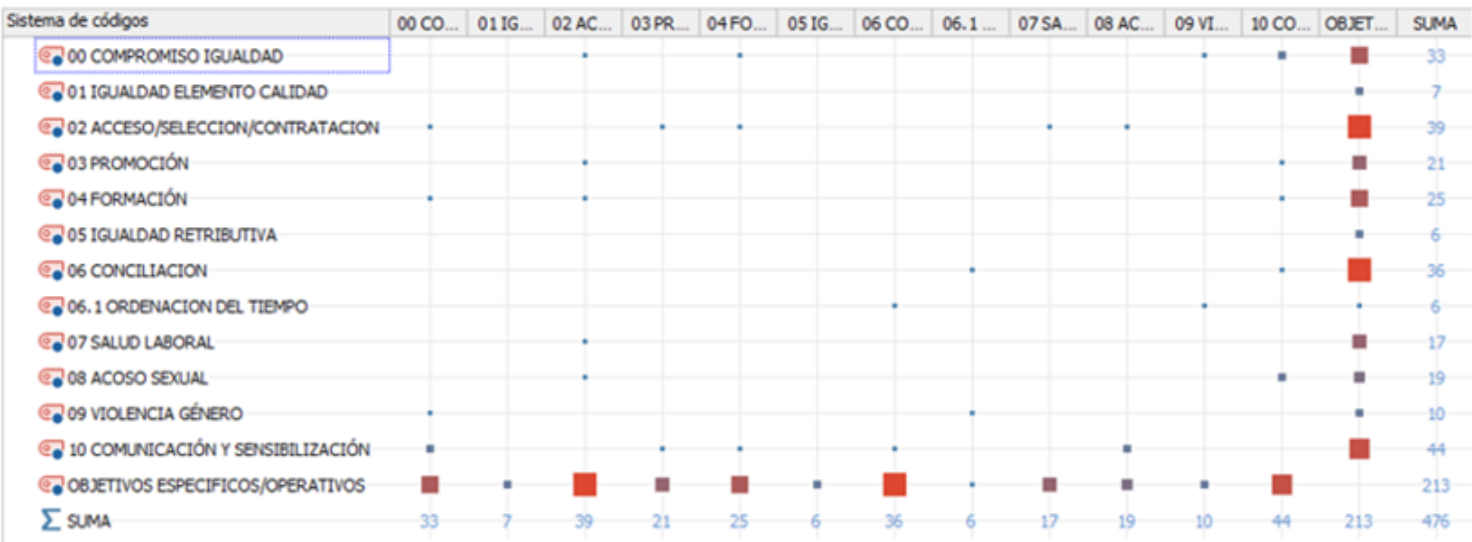

Nota: elaboración propia 
Cuando el analisis (tabla 5) se concreta en los focos de los objetivos especificos, la tabla de coocurrencia de códigos, sigue la misma tendencia que la presentada entre la coocurrencia entre objetivos generales y específicos: la Comunicación y Sensibilización (44), pero se incorporan entre los objetivos especificos el acceso, selección y contratación (39) y la conciliación (36).

A la hora de dar respuesta al objetivo propuesto por esta investigación, se realizó un analisis de coocurrencias entre las diez áreas de los planes y las medidas planteadas. Dicho análisis presenta una imagen de los planes muy propositiva con menor concreción de medidas e indicadores para las Áreas señaladas. La codificación de objetivos generales (89) y de objetivos especificos/operativos (242) contrasta con la menor representación de medidas (136) e indicadores de cumplimiento/resultado (147).

Del contraste entre las diez areas del Plan y el peso de las medidas en dichas areas, la visualización de la coocurrencia de códigos presenta (tabla 6) unos Planes centrados en las medidas de conciliación y en aquellas medidas para la mejora de la comunicación y sensibilización.

\section{Tabla 6}

Visualización de coocurrencia de códigos de áreas de igualdad y medidas a implantar

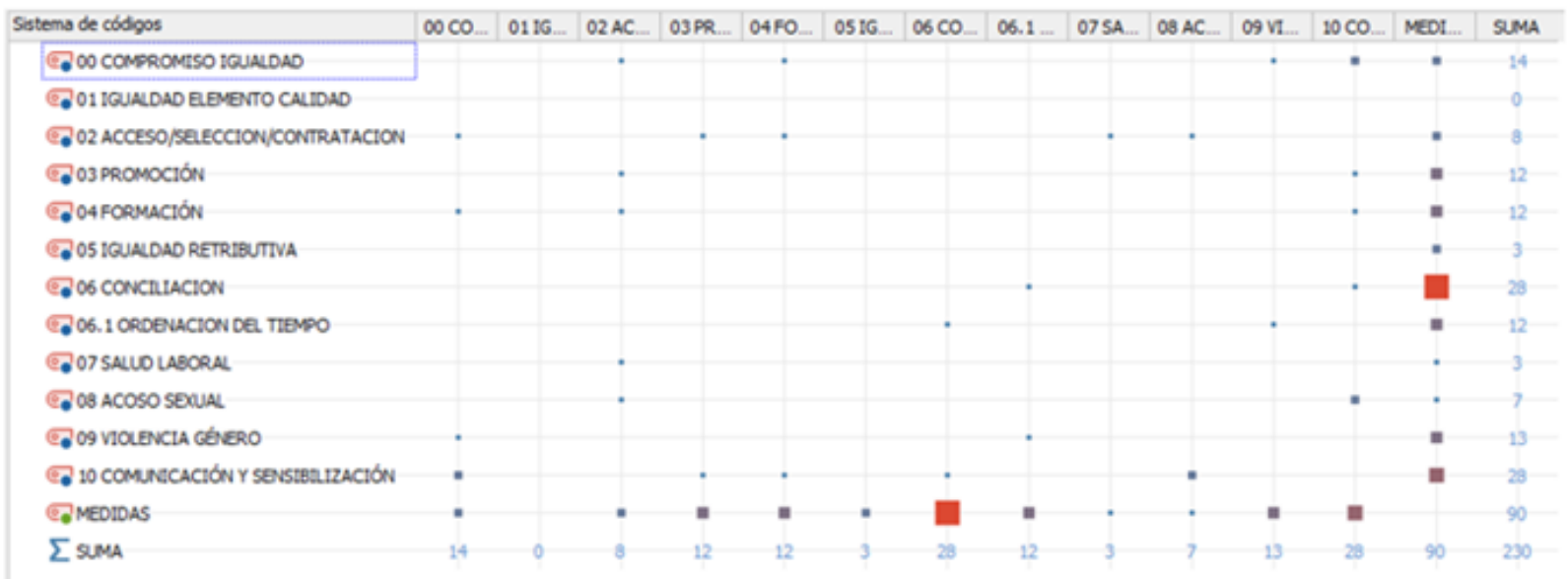

Nota: elaboración propia

\section{Resultado 3: Planes sin concrecion temporal y de recursos}

El Diseño del Plan es una buena fotografía de la potencial eficacia del Plan y al poner el foco en los indicadores se trata de ver aquellos elementos vinculados al desarrollo y aplicación del plan de Igualdad. El tipo de indicadores que presentan los planes son indicadores de seguimiento de las medidas, a excepción de dos de las entidades analizadas. Los indicadores de seguimiento garantizan la coherencia entre los objetivos perseguidos, las medidas adoptadas para su consecución y los criterios de medición. La ausencia de este tipo de indicadores indica que el plan adolece de un "sesgo" no intencionado.

Un segundo momento del análisis, al tratar de ver la potencial efectividad de los planes, resulta llamativo que dos de los seis planes analizados no dispongan de cronograma (tabla 7) para la temporalización de sus acciones y además únicamente dos de los seis establezcan un presupuesto para las medidas planteadas. La efectividad de los planes queda mermada si no se establecen los plazos de consecución de los objetivos o se presupuestan los costes de acciones tan sencillas como la generación de informes desagregados por sexo, cambios en la comunicación interna, campañas de sensibilización en determinadas fechas, etc. 
Tabla 7

Valoración de la potencial efectividad de los planes (Viabilidad- probabilidad de éxito)

\begin{tabular}{|c|c|c|c|c|c|c|c|}
\hline & $\begin{array}{c}\text { Peso } \\
\text { codificación } \\
\text { general } \\
\end{array}$ & $\begin{array}{c}\text { Indicadores } \\
\text { evolución } \\
\text { diagnóstico } \\
\end{array}$ & $\begin{array}{c}\text { Objetivos } \\
\text { generales } \\
\text { /específicos } \\
\end{array}$ & $\begin{array}{c}\text { Indicadores } \\
\text { seguimiento } \\
\text { medidas } \\
\end{array}$ & $\begin{array}{c}\text { Asignación } \\
\text { responsable } \\
\text { departamento }\end{array}$ & Cronograma & Presupuesto \\
\hline $\begin{array}{c}\text { TIGER - } \\
\text { BROTHERS }\end{array}$ & 123 & NO & sí & sí & sí & NO & NO \\
\hline LION- KITS & 100 & NO & sí & NO & NO & NO & NO \\
\hline $\begin{array}{c}\text { JAGUAR - } \\
\text { TIME }\end{array}$ & 109 & NO & sí & NO & sí & sí & sí \\
\hline $\begin{array}{l}\text { PANTHER - } \\
\text { ZONE }\end{array}$ & 457 & NO & sí & sí & sí & sí & NO \\
\hline $\begin{array}{c}\text { ELEPHANT } \\
\text {-ARTIST }\end{array}$ & 152 & NO & sí & sí & sí & sí & NO \\
\hline $\begin{array}{c}\text { DOLPHIN } \\
\text { TECH }\end{array}$ & 66 & NO & sí & sí & sí & sí & sí \\
\hline
\end{tabular}

Nota: elaboración propia

En los Planes analizados se percibe una fuerte apuesta por parte de la Dirección a la hora de plantear objetivos y medidas en pos de la igualdad en la empresa. Sin embargo, la escasa asignación de recursos humanos y económicos para la consecución de dichas medidas provoca lo que Faisal (2011) describe como un vaciado del contenido de los Planes de Igualdad. Para que la implantación de un objetivo funcione en una empresa, es necesario dotar a la acción de personas que lo impulsen y de un presupuesto asignado para su ejecución. En el mejor de los casos ( 2 de los 6 casos analizados), se libera a una persona en la empresa para que impulse el plan, pero no se le asignan recursos, por lo que el margen de actuación es muy limitado, y los resultados insuficientes. En otros casos ni siquiera se libera a nadie para que lidere el proceso en la empresa, sino que convive con otras acciones de la empresa, limitándose una vez más el resultado.

Obviamente esto exige del establecimiento de un control y seguimiento de las acciones que debe estar priorizado y contabilizado en su dimensión económica. En ninguno de los casos analizados, existen indicadores de resultado, lo que informarían sobre el grado esperado de ejecución de cada medida y del plan en su conjunto, así como el número de personas afectadas por las medidas. Tampoco se han encontrado indicadores de impacto que estimen los cambios en términos de igualdad en la empresa como consecuencia de la aplicación de esas medidas concretas.

\section{Discusión}

Según los resultados aportados por esta investigación, la obligatoriedad de los Planes de Igualdad ha supuesto llevar a las empresas el debate de la igualdad que hasta entonces se circunscribía al plano político y académico. La entrada en vigor del Real Decreto Real Decreto-Ley 6/2019, de 1 de marzo, publicado el pasado mes de abril de 2021, obligó a todas las empresas con una plantilla superior a 150 empleados a diseñar e implantar un Plan de Igualdad en la empresa para marzo de 2020, las que tenían más de 100 empleados para marzo de 2021 y las que cuentan con más de 50 empleados para marzo de 2022. Este Real Decreto, que pretende la igualdad en las empresas, se está desarrollando, si bien es interés de esta investigación conocer cómo están actuando las empresas, en qué medida se está implantando y qué efectos se están produciendo.

Es resaltable que todos los planes examinados presenten un programa de trabajo hacia la igualdad, aunque no todos parten de un diagnóstico sólido, puesto que se basan en indicadores desagregados por sexo de la distribución de la plantilla sin entrar en indicadores que aborden en profundidad la existencia de barreras para 
la igualdad en la empresa. Es relativamente sencillo presentar un plan que no entre en profundidad o no plantee cambios relevantes en lo que a igualdad respecta, pero también es cierto que pueden acabar siendo un "cumplir" con la normativa vigente sin entrar en batallas mayores. En este sentido se centran en lo más fácil, el diseño, que luego no viene acompañado de la acción.

A la ya mencionada escasa asignación de responsabilidad en las medidas a implantar existe en todos ellos un déficit participativo que queda relegado a una comisión y la participación de algunos empleados en dinámicas cualitativas de escaso rigor metodológico. Lógicamente entrar en dinámicas más exigentes para la empresa lastra el impulso de acciones concretas que puedan dar pasos en este sentido.

La mayoría de las empresas se encuentran en el desarrollo del II Plan sin haber realizado una evaluación de los objetivos generales y específicos y medidas planteadas en los planes previos, lo que dificulta ver la evolución y el impacto de las medidas implantadas en la empresa. Da la impresión de que se avanza, siguiendo los requerimientos y cumpliéndolos de cara a una inspección, pero sin realizar ninguna acción de calado, nada que pueda generar ruido dentro de la organización.

La apuesta por objetivos y medidas orientados a la comunicación describen a unas empresas en un incipiente estado de preocupación por la igualdad, pero no tan fuerte como para obligarles a cambiar. Dan cumplimiento a la normativa, sin entrar en cambios sustanciales. Centran los esfuerzos en una comunicación interna (que los empleados tengan presente que nuestra empresa está por la igualdad) e incluso externa, algo relativamente rápido y fácil. Obviamente la viabilidad de medidas orientadas a la comunicación y sensibilización de medidas es mucho más factible que plantear medidas sobre el acceso y selección que eviten sesgos de partida o medidas para la promoción de mujeres a posiciones intermedias o directivas.

Son conscientes de la necesidad de mejora en la selección, pero en general, no llegan a concretar medidas con esta orientación. Igualmente sucede con la promoción, barrera fundamental para la igualdad en las empresas (Martín de la Torre, 2017; Baños, 2018; Hoops et al., 2018; Silver, 2019). Los criterios claros de selección en igualdad de oportunidades, una transparencia en la retribución de los empleados y criterios claros de promoción, son el punto débil de estas empresas en lo que a igualdad se refiere (Salinas, y Bagni, 2017; Correll, 2017; Trotter et al., 2017; Morgan, 2019; Bennedsen et al., 2019; Bryant, 2017). Hay un claro impulso de la comunicación en detrimento de la promoción. Se puede trabajar en la entrada de las personas a la empresa, en una no discriminación que posibilite una igualdad de partida, pero si luego no va acompañada de una revisión paritaria de los procesos de promoción, el problema sigue estando dentro de la empresa. Hay ya empresas que han dado un paso al frente, incorporando ejemplos de buenas prácticas para la igualdad en la empresa (PwC, 2020). Quieren y dan pasos para favorecer la incorporación de más mujeres a la empresa. Sin embargo, en la promoción se observa una inclinación de la balanza hacia el género masculino (30/70), produciendo este "efecto tijera" analizado en la Academia (Martínez Ruiz, Merma-Molina, y Ávalos Ramos, 2018): entran más mujeres, pero promocionan más hombres.

En los planes analizados, no hay ni una sola mención a la brecha de género salarial. Este es un nuevo reto para los Planes de Igualdad puesto que, desde el 14 de abril de 2021, todas las empresas deberán contar con un registro adaptado al RD 902/2020. Esta nueva exigencia aborda la brecha de género gracias a los indicadores de complementos salariales y las percepciones extra salariales, donde la fotografía sobre la desigualdad es nítida.

Para finalizar esta discusión indicando que las empresas orientan la mejora hacia áreas en las que no van a tener dificultades y donde pueden obtener metas a corto plazo, sin que conlleve un gran esfuerzo a la organización. Utilizan los planes de igualdad como instrumentos para la sensibilización y comunicación, si bien existe un escaso foco en las áreas importantes: selección y promoción. Estos últimos más complicados, por los cambios que le 
suponen a la propia organización. Navegan por aguas tranquilas y evitan las zozobras que producen las aguas revueltas.

\section{Conclusiones}

La incorporación de la mujer al trabajo ha experimentado un impulso a finales de los años ochenta del siglo pasado. Esta creciente incorporación no ha sido en igualdad de condiciones. Numerosos estudios ponen de manifiesto que existe una cultura masculina en las empresas que dificulta la entrada y el ascenso profesional de las mujeres. Los fenómenos del techo de cristal o el suelo pegajoso, entre otros, evidencian las dificultades que tienen las mujeres para poder competir en igualdad de oportunidades en las empresas. Aún en las empresas donde hay una práctica en pos de la igualdad, ésta se reduce cuando se asciende en la jerarquía de la empresa, siendo los puestos de dirección (CEO y Director General) ocupados mayoritariamente por hombres. Al analizar los Consejos de dirección de las empresas, un $27,7 \%$ de los puestos de administración de las empresas del IBEX 35 (INE,2021) dista todavía de la ansiada igualdad, lo que supone una pérdida de talento femenino en las empresas, fruto de ese desequilibrio que se observa en la realidad empresarial.

Ante esta realidad, el Gobierno de España ha definido varias normativas, leyes y Reales Decretos con el objetivo de lograr normalizar la presencia de la mujer en las empresas de nuestro país y evidenciar su claro compromiso con la igualdad. Esto obliga a las empresas a un cumplimiento de la Ley, para no verse expuestas a la correspondiente sanción, a la vez que buscan ofrecer externa e internamente una imagen moderna de empresa que fomenta la igualdad de género. Son los Planes de Igualdad de las empresas, el instrumento con el que cuentan y que tienen que implantar, la declaración de las empresas, donde manifiestan sus objetivos y metas alcanzables a corto plazo y su compromiso por la igualdad.

En las empresas participantes en el estudio, compuesto por miembros de un Grupo-Motor que impulsa la igualdad de la mujer en la empresa, hemos sometido a análisis sus Planes de Igualdad, encontrando principalmente acciones orientadas a conseguir una mejor comunicación interna y externa de a igualdad, buscando una sensibilización de los empleados de la empresa y los stakeholders. En definitiva, generar una conciencia, una preocupación e interés por el tema. Los planes analizados centran en medidas de conciliación, en comunicación y en sensibilización en materia de igualdad que como punto de partida es importante. Esto obedece a la facilidad de cumplir con estos objetivos, que no implican grandes cambios ni transformaciones en las empresas.

Sin embargo, no entran en temas que son los que pueden producir un cambio real en la igualdad dentro de la empresa: la política y criterios de selección, la promoción interna y sus oportunidades dentro de la empresa o la remuneración. Estos criterios constituyen los elementos clave en materia real de igualdad en la empresa y son los que se echan de menos, los que no se acometen. Claramente, son temas más delicados y difíciles de llevar a cabo por la dirección, por lo que no se abordan en la empresa. En este sentido observamos una clara incongruencia en los objetivos generales y específicos con respecto a las medidas reales adoptadas.

Finalmente, en la valoración del potencial de los planes de estas empresas, vemos que no hay indicadores de evolución de los diagnósticos realizados (por lo que no se pueden comprobar los avances, si es que se dan), faltan indicadores de seguimiento y, en algún caso un responsable directo; a esto hay que añadir la falta mayoritaria de un presupuesto para poder desarrollar las acciones ni un cronograma, quedando mermada la efectividad de los planes. Las empresas tienen que empezar a tomar conciencia que los Planes de Igualdad han llegado y las exigencias van a ir en aumento.

Podemos concluir que los Planes de Igualdad de las empresas analizadas evidencian que cumplen con lo que les resulta más factible, pero permanecen alejados de lo entraña más dificultad. Acometen lo asumible y evitan lo 
complicado. Cumplen con una buena imagen, porque están haciendo esfuerzos, pero éstos no son suficientes para poder hablar de un cambio positivo en la carrera de igualdad de la mujer en la empresa.

Entre las limitaciones del estudio podemos mencionar que si bien es un numero exiguo de planes, éstos pertenecen a empresas importantes que, además, forman parte de un grupo-motor por la igualdad en las empresas. Nos ilustran una realidad a la que podemos estar sometidos: estamos viviendo cambios provocados por las nuevas leyes, pero como todos los cambios son lentos y están enfocados más a mostrar una imagen favorable de la empresa que a evidenciar la realidad: es la filosofía de comenzar por lo fácil y dejamos lo difícil. Y la realidad jurídica y social comienza a presionar.

Queda todavía camino por andar en la igualdad de las empresas. Son muchas las prioridades a las que se enfrenta el mundo empresarial y la igualdad, a golpe de decreto y Ley, se hace un hueco en la lista y hay que darle una respuesta. Esto no garantiza que las empresas cambien sus políticas internas para favorecer una igualdad real, sino que, de momento, abordan lo que es factible y tiene buena prensa. Nadie dijo que esto iba a ser un camino fácil, y los resultados del estudio así lo demuestran, pero tampoco podemos aventurar en qué tramo del camino estamos. No es suficiente una Ley o un Real Decreto para cambiar una realidad instaurada desde hace mucho tiempo. Es un paso adelante, pero hace falta que la empresa avance en este tema, sabiendo que la incorporación de talento femenino a la empresa se va a traducir en beneficio para la misma. Sólo si la empresa toma conciencia de ello se producirá el cambio.

\section{Bibliografía}

Baños, C. C. (2018). El techo de cristal en el sector público: Acceso y promoción de las mujeres a los puestos de responsabilidad. RES. Revista Española de Sociología, 27(3), 475-489.

Barberá Heredia, E., Ramos, A., Sarrió, M., \& Candela, C. (2002). Más allá del" techo de cristal". Diversidad de género. Revista del ministerio de trabajo y asuntos sociales, 40, 55-68.

Begum, A. N., \& Brindha, G. (2017). Challenges and Strategies for Retaining Women Talent in it Industry. Internat ional Journal of Civil Engineering and Technology, 8(10).

Belkin, L. (2003). The opt-out revolution. New York.

Beltrán, A.; Martínez, M. y Noguera, A. (2018). Women Matter 2017: a way forward for Spain. https://www.mckinsey.com/ /media/McKinsey/Featured\%20Insights/Europe/Women\%20matter\%20201 7\%20A\%20way\%20forward\%20for\%20Spain/Women-matter-2017-A-way-forward-for-Spain.PDF

Bennedsen, M., Simintzi, E., Tsoutsoura, M., \& Wolfenzon, D. (2019). Do firms respond to gender pay gap transparency? (No. w25435). National Bureau of Economic Research.

Biedma-Ferrer, J. M. (2017). La mujer directiva. La presencia de la mujer en los Consejos de Administración de las Compañías del IBEX 35.

Bieto, E. y Cauqui, P. (21 de abril de 2021). Esade Gender Monitor 2020. https://itemsweb.esade.edu/wi/Prensa/Esade_Gender_Monitor_2020.pdf

Bourdieu, P., \& Distinction, L. (1979). Sociale du Jugement. Les Editions de Minuit.

Bortz, D. (16 de abril de 2021). Can blind hiring improve workplace diversity? https://www.shrm.org/hrtoday/news/hr-magazine/0418/Pages/can-blind-hiring-improve-workplace-diversity.aspx

Bryant, L. D., Burkinshaw, P., House, A. O., West, R. M., \& Ward, V. (2017). Good practice or positive action? Using $Q$ methodology to identify competing views on improving gender equality in academic medicine. BMJ open, 7(8), e015973. 
Cancio, F. (8 de marzo de 2021). ¿Cuántas mujeres hay en las Fuerzas Armadas? La Razón. https://www.larazon.es/espana/20210308/xdl6xotr5nczln5gbvwhsrtsgy.html

Cebrián, I., \& Moreno, G. (2008). La situación de las mujeres en el mercado de trabajo español: desajustes y retos. Economía industrial, (367), 121-137.

Chinchilla, N., \& León, C. (2007). Guía de buenas prácticas de la empresa flexible. Comunidad de Madrid.

ClosingGap (2020). La mujer como referente en la transformación social hacia un consumo más responsable. https://closingap.com/wp-content/uploads/2020/11/CG-Informe-Consumo-y-Mujer.pdf

CNMV (21 de abril 2021). http://www.cnmv.es/Portal/verDoc.axd?t=\%7B8e231ab6-4d7a-4617-98d4ff41b22aca64\%7D

Collins, G. (2005). The gendered nature of mergers. Gender, Work \& Organization, 12(3), 270-290.

Correll, S. J. (2017). SWS 2016 Feminist Lecture: Reducing gender biases in modern workplaces: A small wins approach to organizational change. Gender \& Society, 31(6), 725-750.

Dazn (14 de abril 2021). Mujeres y baloncesto. https://www.dazn.com/esES/home/Articleld:cvtadeojmu9l1f390ebzsd8e5

De España, G. (2007). Ley Orgánica 3/2007, de 22 de marzo, para la igualdad efectiva de mujeres y hombres. Boletín oficial del Estado, 71, 12611-12645.

De España, G. (2005). Orden PRE/525/2005, de 7 de marzo, por la que se da publicidad al Acuerdo de Consejo de Ministros por el que se adoptan medidas para favorecer la igualdad entre mujeres y hombres.

De España, G. (2005). Orden APU/526/2005, de 7 de marzo, por la que se dispone la publicación del Acuerdo de Consejo de Ministros de 4 de marzo de 2005, por el que se aprueba el Plan para la igualdad de género en la Administración General del Estado.

De los Trabajadores, E. (2015). Real Decreto Legislativo 2/2015, de 23 de octubre, por el que se aprueba el texto refundido de la Ley del Estatuto de los Trabajadores. Boletín Oficial del Estado, (255).

Del Estado, J. (2019). Real Decreto-ley 6/2019, de 1 de marzo, de medidas urgentes para garantía de la igualdad de trato y de oportunidades entre mujeres y hombres en el empleo y la ocupación.

De Miguel, R. (10 de abril de 2021). Una leyenda femenina en el Grand National. El País. https://elpais.com/deportes/2021-04-10/rachel-blackmole-se-convierte-en-la-primera-mujer-en-ganar-elgrand-national.html

Díaz, T. (2 de abril de 2021). Las mujeres emiten un 10\% menos de CO2 que los hombres por sus hábitos. El Economista. Las mujeres emiten un 10\% menos de CO2 que los hombres por sus hábitos - elEconomista.es

Diez, E. (3 de febrero de 2021). Año 2021: La comunidad internacional no logra la igualdad entre hombres y mujeres. The Conversation. https://theconversation.com/ano-2021-la-comunidad-internacional-no-lograla-igualdad-entre-hombres-y-mujeres-154024

Dobbin, F., \& Kelly, E. L. (2007). How to stop harassment: Professional construction of legal compliance in organizations. American Journal of Sociology, 112(4), 1203-1243.

Domínguez, B. (3 de enero de 2018). Islandia da un paso más en equidad y acaba con la brecha salarial. El País. https://elpais.com/internacional/2018/01/03/actualidad/1514990392_124251.html 
Doldor, E., Vinnicombe, S., Gaughan, M., \& Sealy, R. (2012). Gender diversity on boards: The appointment process and the role of executive search firms. Equality and human rights commission research report, 85, 1-98.

Eagly, A. H. (1987). Reporting sex differences.

Edgerton, J.D., \& Roberts, L. (2014). Cultural capital or habitus? Bourdieu and beyond in the explanation of enduring educational inequality. Theory and Research in Education, 12, 193 - 220.

Escapa, R., \& Ten, L. M. (2010). Estrategias de liderazgo para mujeres directivas. Generalitat de Catalunya, Departament de Treball.

Escot Mangas, L., Fernández Cornejo, J. A., Albert López-Ibor, R., \& Samamed Rodríguez, M. (2008). Un experimento de campo para analizar la discriminación contra la mujer en los procesos de selección de personal.

Faisal, F. (2011). Public Policy and Gender Mainstreaming Strategy: Redressing Gender Inequality. Interdisciplinaty Journal of Research in Business, 1(7), 8-16.

Fajak, A., \& Haslam, S. A. (1998). Gender solidarity in hierarchical organizations. British Journal of Social Psychology, 37(1), 73-94.

Fundació Factor Humá (2010). Mujer y liderazgo. https://factorhuma.org/es/unidades-de-conocimientoblog/8259-mujer-y-liderazgo

Ferro Veiga, J. M. (2013). Aspectos formales y materiales del acoso laboral y de la violencia de género e intrafamiliar. Aspectos formales y materiales del acoso laboral y de la violencia de género e intrafamiliar, $0-0$.

Gallego-Álvarez, I., García-Sánchez, I. M., \& Rodríguez-Dominguez, L. (2010). The influence of gender diversity on corporate performance: La influencia de la diversidad de género en el rendimiento empresarial. Revista de Contabilidad-Spanish Accounting Review, 13(1), 53-88.

García-Ael, C., Cuadrado, I., \& Molero, F. (2012). Think-manager-Think-male vs. Teoría del Rol Social: ¿cómo percibimos a hombres y mujeres en el mundo laboral? Estudios de psicología, 33(3), 347-357.

Gelambi- Torrell (2015): Los Planes de Igualdad como instrumento para lograr la igualdad de género en América Latina: ¿Algo más que un objetivo? Gender Equality Programmes as mechanisms to achieve gender equality in Latin America. Is it just a goal? Científico Sapiens Research Vol. 5(1)-2015 / pp: 43-49 / ISSN-e: 2215-9312 Sapiens Research Group

Goyette, K. A., \& Mullen, A. L. (2006). Who studies the arts and sciences? Social background and the choice and consequences of undergraduate field of study. The Journal of Higher Education, 77(3), 497-538.

Hacohen, R., Mottershaw, A. y Meer, J. (8 de marzo de 2021). Why is more likely to apply for flexible Jobs men or women? The Behavioral Insights team. https://www.bi.team/blogs/who-is-more-likely-to-apply-forflexible-jobs-men-or-women/

Hewlett, S. A. y Luce, C. B. (2005). Off-ramps and on-ramps: keeping talented women on the road to success. Harvard Business Review, 83(3), 43

Hoops, H. E., Brasel, K. J., Dewey, E., Rodgers, S., Merrill, J., Hunter, J. G., \& Azarow, K. S. (2018). Analysis of gender-based differences in surgery faculty compensation, promotion, and retention: establishing equity. Annals of surgery, 268(3), 479-487.

Hopkins, M. M., \& O'Neil, D. A. (2007). Women and success: dilemmas and opportunities. Handbook on women in business and management, 132-153. 
Huang, J., Krivkovich, A., Starikova, I., Yee, L., \& Zanoschi, D. (2019). Women in the Workplace 2019. San Francisco: Retrieved from McKinsey \& Co. website: https://www. mckinsey. com/featured-insights/genderequality/women-in-the-workplace.

Hunt, V., Prince, S., Dixon-Fyle, S., \& Yee, L. (2018). Delivering through diversity. McKinsey \& Company, 231.

Kendall, V. E. (2018). Identifying Factors That Impact Female Leadership Presence.

ILO (2019). Breaking barriers: Unconscious gender bias in the workplace. https://www.ilo.org/wcmsp5/groups/public/---ed_dialogue/--act_emp/documents/publication/wcms_601276.pdf

INE (21 de abril de 2021). https://www.ine.es/ss/Satellite?L=es_ES\&c=INESeccion_C\&cid=1259925595694\&p=1254735110672\&pag ename=ProductosYServicios\%2FPYSLayout

Inga, S. M., \& Tristán, O. M. (2020). ¿Por qué hay pocas mujeres científicas? Una revisión de literatura sobre la brecha de género en carreras STEM. aDResearch: Revista Internacional de Investigación en Comunicación, (22), 118-133.

Kaiser, R. B., \& Wallace, W. T. (2016). Gender bias and substantive differences in ratings of leadership behavior: Toward a new narrative. Consulting Psychology Journal: Practice and Research, 68(1), 72.

Kellerman, B., \& Rhode, D. L. (2007). Women and leadership: The state of play and strategies for change (Vol. 141). Jossey-Bass.

Kersley, R., Klerk, E., Boussie, A., Sezer Longworth, B., Anamootoo Natzkoff, J., \& Ramji, D. (2019). The CS Gender 3000 in 2019: the Changing Face of Companies. Research Institute, Credit Suisse.

Khalife, D., \& Chalouhi, A. (2013). Gender and business performance. International Strategic Management Review, 1(1-2), 1-10.

Krivkovich, A., Kutcher, E., \& Yee, L. (2016). Breaking down the gender challenge. McKinsey Quarterly, 2, 8-13.

Lerer, Z., \& Ben-Eliyahu, H. (2019). Handbook of organizational gender consultantion and intervention. Heinrich Boell Stiftung https://il.boell.org/sites/default/files/handbook_of_gender_consultation_and_intervention_2.pdf

Loayza, N. y Trumbic, T. (23 de febrero de 2021). Women, Business and the Law 2021: Women's economic empowerment is critical to resilient recovery efforts. World Bank Blogs.

https://blogs.worldbank.org/developmenttalk/women-business-and-law-2021-womens-economicempowerment-critical-resilient-recovery

López-Ibor, R. A. (2008). Análisis de la presencia de las mujeres en los puestos directivos de las empresas madrileñas. Consejo Económico y Social, Comunidad de Madrid.

Llobet, C. V. (2006). Mujeres invisibles (Vol. 162). Random House Mondadori.

Martín de la Torre, M. B. (2017). Derechos de conciliación y promoción profesional de la mujer.

Martínez Ruiz, M. Á., Merma-Molina, G., \& Ávalos Ramos, M. A. (2018). La brecha de género en la academia universitaria: inequidad de oportunidades de participación, capacitación y promoción.

McKinney, V. R., Wilson, D. D., Brooks, N., O'Leary-Kelly, A., \& Hardgrave, B. (2008). Women and men in the IT profession. Communications of the ACM, 51(2), 81-84. 
McKinsey \& Company (2015). A Ceo's guide to gender equality. https://www.mckinsey.com/featuredinsights/leadership/a-ceos-guide-to-gender-equality

Maitland, A. (2015). El cambio de poder en las relaciones de género está en marcha. En Reinventar la empresa en la era digital (pgs. 195-215). BBVA.

Martín, T. T. (2005). De la imposible conciliación a los permanentes malos arreglos. Cuadernos de relaciones laborales, 23(1), 015-033.

McKinsey, C. (2007). Women matter: Gender diversity. A Corporate Performance Driver, 1-13.

Ministerio de Asuntos Exteriores y de Cooperación (21 de abril de 2021). Consejo de Europa: Estrategia de Igualdad de Género 2018-2023. https://rm.coe.int/estrategia-de-igualdad-de-genero-del-coe-esmsg/16808ac960Una

Ministerio de Igualdad (18 de abril de 2021). Guia para la elaboración de planes de igualdad en las empresas. https://www.igualdadenlaempresa.es/asesoramiento/diagnostico/docs/Guia_pdi.pdf

Ministerio de Sanidad, Servicios sociales e Igualdad (2016). Liderazgo femenino. Boletin Igualdad Empresa XXIX. https://www.igualdadenlaempresa.es/actualidad/boletin/docs/Boletin_Igualdad_en_la_Empresa_BIE_29. pdf

Molero, F., Cuadrado, I., García-Ael, C., Recio, P., \& Rueda, B. (2009). Mujer y liderazgo en el siglo XXI: Una aproximación psicosocial a los factores que dificultan el acceso de la mujer a los puestos de responsabilidad. Madrid: Instituto de la Mujer.

Morgan, J. A. (2019). How to Close the Gender Pay Gap: Transparency in Data regarding Compensation Is the Key. Conn. J. Int'l L., 35, 407.

Munduate, L. (2003). Género y liderazgo. Diferencias entre hombres y mujeres en el acceso a los puestos directivos. Revista de psicología Social, 18(3), 309-314.

Nieto, S. (10 de abril de 2021). Lisa Keetley, general de brigada y fundadora de la Red de Mujeres Militares: "Se puede ser militar y feminista, absolutamente sí". El Mundo. https://www.elmundo.es/yodona/mujeresinfluyentes/2021/04/10/606c811221efa0b8458b4629.html

Nieto, S. A., \& Hernández, P. M. (2007). Factores que dificultan el acceso de las mujeres a puestos de responsabilidad: una revisión teórica. Apuntes de psicología, 25(2), 201-214.

OIT (2019). Las mujeres en la gestión empresarial. Argumento para un cambio. https://www.ilo.org/wcmsp5/groups/public/---dgreports/---dcomm/--publ/documents/publication/wcms_700977.pdf

Peluso, M., Baird C. y Kesterson-Townes, L. (2019). Women, leadership and the priority paradox. IBM Institute for business value.

PwC (2020). Inspirando. Casos de éxito en diversidad de género. Recuperado de https://www.pwc.es/es/publicaciones/gestion-empresarial/assets/mujer-directiva-inspirando.pdf

PriceWaterhouseCoopers UK (2021). Women in work index 2021. https://www.pwc.co.uk/services/economics/insights/women-in-work-index.html

Real Academia de la Historia (12 de diciembre de 2014). Carmen Iglesias Cano elegida directora de la Real Academia de la Historia. https://www.rah.es/dona-carmen-iglesias-cano-elegida-directora-de-la-realacademia-de-la-historia/ 
Ro, C. (2021). Helping workers understand their implicit biases is helpful, in theory - but these programmes are controversial. Who's right? Bbc.com https://www.bbc.com/worklife/article/20210326-the-complicatedbattle-over-unconscious-biastraining\#: :text=Helping\%20workers\%20understand\%20their\%20implicit,Who's\%20right\%3F\&text=Starbu cks\%20and\%20have\%20been\%20doing\%20it.

Robinson, V. (2018). Desigualdades de género: problemas «pasados» y futuras posibilidades. En ¿Hacia una nueva llustración? Una década trascendente. BBVA.

Roksa, J., \& Robinson, K. (2017). Cultural capital and habitus in context: the importance of high school collegegoing culture. British Journal of Sociology of Education, 38, 1230 - 1244.

Salinas, P. C., \& Bagni, C. (2017). Gender equality from a european perspective: myth and reality. Neuron, 96(4), 721-729.

Sarrió, M., Barberá, E., Ramos, A., \& Candela, C. (2002). El techo de cristal en la promoción profesional de las mujeres. Revista de psicología social, 17(2), 167-182.

Schein, V. E. (1973). The relationship between sex role stereotypes and requisite management characteristics. Journal of applied psychology, 57(2), 95.

Silver, J. K. (2019). Gender equity on journal editorial boards. The Lancet, 393(10185), 2037-2038.

Suisse, C. (2014). The CS Gender 3000: Women in Senior Management.

Tienari, J., Meriläinen, S., Holgersson, C., \& Bendl, R. (2013). And then there are none: on the exclusion of women in processes of executive search. Gender in Management: An International Journal.

Trotter, R. G., Zacur, S. R., \& Stickney, L. T. (2017). The new age of pay transparency. Business Horizons, 60(4), 529-539.

Turban, S., Wu, D., \& Zhang, L. (2019). When gender diversity makes firms more productive. Harvard Business Review, 11.

Valdebenito, O. G. (2017). Estudios de liderazgo de hombres y mujeres. Revista Política y Estrategia, 126, 1335.

VicHealth Coronavirus Victorian Wellbeing Impact Study (2020). Victorian Health Promotion Foundation. Melbourne (https://doi.org/10.37309/2020.PO909)

Vega, S., de la Concha, J. y Pérez, M. (2007). Estudio de las barreras culturales que dificultan la aplicación de mecanismos de producción flexible en las empresas, https://www.inmujeres.gob.es/publicacioneselectronicas/documentacion/Documentos/DE0486.PDF

Wagner, H. M. (2011). The bottom line: corporate performance and gender diversity in the c-suite (20042008). Available at SSRN 1980371.

Wajcman, J. (2006). Technocapitalism meets technofeminism: women and technology in a wireless world. Labour \& Industry: a journal of the social and economic relations of work, 16(3), 7-20. Wajcman, J. (2006). Technocapitalism meets technofeminism: women and technology in a wireless world. Labour \& Industry: a journal of the social and economic relations of work, 16(3), 7-20.

Wallis, C. (2004). The case for staying home. Time, 163(12), 51-51.

Wilson, A., \& Urick, A. (2021). Cultural Reproduction Theory and Schooling: The Relationship between Student Capital and Opportunity to Learn. American Journal of Education, 127, 193-232. 
World Bank Group. (2019). Women, business and the law 2019: A decade of reform. World Bank.

World Economic Forum (30-de marzo de 2021). Global Gender Gap Report 2021. https://www.weforum.org/reports/global-gender-gap-report-2021

Zhang, L. (2020). An institutional approach to gender diversity and firm performance. Organization Science, 31(2), 439-457.

Esta obra está bajo una Licencia Creative Commons

Attribución-NoCommercial 4.0 International

$(\mathrm{cc})$ BY-NC 